NONCOMMUTATIVE HARMONIC ANALYSIS

WITH APPLICATIONS TO PROBABILITY III

BANACH CENTER PUBLICATIONS, VOLUME 96

INSTITUTE OF MATHEMATICS

POLISH ACADEMY OF SCIENCES

WARSZAWA 2012

\title{
REMARKS ON CATALAN AND SUPER-CATALAN NUMBERS
}

\author{
ANNA DOROTA KRYSTEK and ŁUKASZ JAN WOJAKOWSKI \\ Mathematical Institute, University of Wroctaw \\ pl.Grunwaldzki 2/4, 50-384 Wroctaw, Poland \\ E-mail: Anna.Krystek@math.uni.wroc.pl,Lukasz.Wojakowski@math.uni.wroc.pl
}

\begin{abstract}
In this article we discuss the Catalan and super-Catalan (or Schröder) numbers. We start with some combinatorial interpretations of those numbers. We study two probability measures in the context of free probability, one whose moments are super-Catalan, and another, whose even moments are super-Catalan and odd moments are zero. With the use of the latter we also show some new formulae for evaluation of the Catalans in terms of super-Catalans and vice-versa.
\end{abstract}

1. Positive definite sequences as moments of probability measures. Assume that we have a positive definite sequence $a_{n}$ with $a_{0}=1$. It is known [Ch that there exist one (or many) positive probability measures $\mu$ on the real line such that

$$
a_{n}=\int_{\mathbb{R}} x^{k} \mathrm{~d} \mu(x)=m_{\mu}(n) .
$$

In the present paper we shall investigate the sequence of the so-called super-Catalan numbers. We will be interested in their properties and relation to the ordinary Catalan numbers, as well as in the properties of the associated probability measures. Let us now introduce some notation that will be useful later.

The generating function of moments of a compactly supported probability measure $\xi$ will be denoted by

$$
M_{\xi}(z)=\sum_{n=0}^{\infty} m_{\xi}(n) z^{n},
$$

for $z$ in some neighbourhood of zero. The Cauchy transform of $\xi$ is given by

$$
G_{\xi}(z)=\int_{\mathbb{R}} \frac{\mathrm{d} \xi(x)}{z-x},
$$

2010 Mathematics Subject Classification: 46L53, 46L54, 60E10.

Key words and phrases: Catalan numbers, super-Catalan numbers, partitions.

Partially sponsored with KBN grant no N N201 364436.

The paper is in final form and no version of it will be published elsewhere. 
for $z$ in a neighbourhood of infinity, and it is related to $M_{\xi}(z)$ by

$$
G_{\xi}(z)=\frac{1}{z} M_{\xi}\left(\frac{1}{z}\right) .
$$

By a theorem of Stieltjes, see $[\mathrm{Ak}$, the Cauchy transform of a probability measure $\xi$ with moments of all orders can be expressed as a formal continued fraction:

$$
G_{\xi}(z)=\frac{1}{z-\alpha_{0}-\frac{\lambda_{0}}{z-\alpha_{1}-\frac{\lambda_{1}}{z-\alpha_{2}-\frac{\lambda_{2}}{\ddots}}} .}
$$

The sequences $\alpha_{n}, \lambda_{n}$ are the Szegö-Jacobi parameters of the recurrence formula for the orthonormal polynomials with respect to $\xi$ :

$$
\begin{aligned}
P_{0}^{\xi}(x) & =1, \quad P_{1}^{\xi}(x)=\frac{x-\alpha_{0}}{\sqrt{\lambda_{0}}}, \\
\left(x-\alpha_{n}\right) P_{n}^{\xi}(x) & =\sqrt{\lambda_{n}} P_{n+1}^{\xi}(x)+\sqrt{\lambda_{n-1}} P_{n-1}^{\xi}(x) .
\end{aligned}
$$

Orthonormality means that

$$
\int P_{n}^{\xi}(x) P_{m}^{\xi}(x) \mathrm{d} \xi(x)=\delta_{n, m}
$$

2. Catalan and super-Catalan numbers. Catalan numbers, named after the Belgian mathematician Eugene Charles Catalan (1814-1894), were first encountered by Leonhard Euler. The numbers are given by

$$
C_{n}=\frac{1}{n+1}\left(\begin{array}{c}
2 n \\
n
\end{array}\right)
$$

and satisfy the recurrence relation

$$
C_{n+1}=\sum_{k=0}^{n} C_{k} C_{n-k}, \quad C_{0}=1,
$$

or equivalently

$$
C_{n+1}=\frac{2(2 n+1)}{n+2} C_{n}, \quad C_{0}=1 .
$$

$C_{n}$ are the even moments of the Wigner measure:

$$
C_{n}=\int_{\mathbb{R}} \frac{1}{2 \pi} \sqrt{4-x^{2}} \mathbb{1}_{[-2,2]}(x) x^{2 n} \mathrm{~d} x .
$$

Super-Catalan numbers were introduced by Friedrich Wilhelm Karl Ernst Schröder (1870) in discussion of four bracketing problems. Their two known defining recurrence relations are:

$$
\mathcal{C}_{0}=\mathcal{C}_{1}=1, \quad \mathcal{C}_{n}=\frac{3(2 n-1) \mathcal{C}_{n-1}-(n-2) \mathcal{C}_{n-2}}{n+1}, \quad n \geq 2,
$$

and

$$
\mathcal{C}_{0}=1, \quad \mathcal{C}_{n}=-\mathcal{C}_{n-1}+2 \sum_{j=0}^{n-1} \mathcal{C}_{j} \mathcal{C}_{n-j-1}, \quad n \geq 1
$$


A closed form expression for $n>1$ in terms of Legendre polynomials $P_{n}(x)$ is

$$
\mathcal{C}_{n}=\frac{3 P_{n-1}(3)-P_{n-2}(3)}{4 n}=\frac{1}{4}\left(-P_{n}(3)+6 P_{n-1}(3)-P_{n-2}(3)\right) \text {. }
$$

The generating function of $\mathcal{C}_{n}$ is

$$
M_{\mu}(x)=\sum_{n=0}^{\infty} \mathcal{C}_{n} x^{n}=\frac{1+x-\sqrt{1-6 x+x^{2}}}{4 x} .
$$

\section{Interpretations of Catalan and super-Catalan numbers}

1. Catalan $C_{n}$ is the number of different ways $n+1$ factors can be completely parenthesized $[\mathrm{St}]$ :

$$
((a b) c) d, \quad(a(b c)) d, \quad(a b)(c d), \quad a((b c) d), \quad a(b(c d)) .
$$

Super-Catalan $\mathcal{C}_{n}$ is the number of different ways $n+1$ factors can be incompletely parenthesized FMP:

$$
\begin{aligned}
& (a b) c d, \quad a(b c) d, \quad a b(c d), \quad(a b)(c d), \quad(a b c) d, \quad a(b c d), \\
& ((a b) c) d, \quad(a(b c)) d, \quad a((b c) d), \quad a(b(c d)), \quad a b c d .
\end{aligned}
$$

2. A rooted binary tree is full if every vertex has either two children or no children. $C_{n}$ is the number of such trees with $2 n+1$ vertices $\mathrm{Ai}$ :
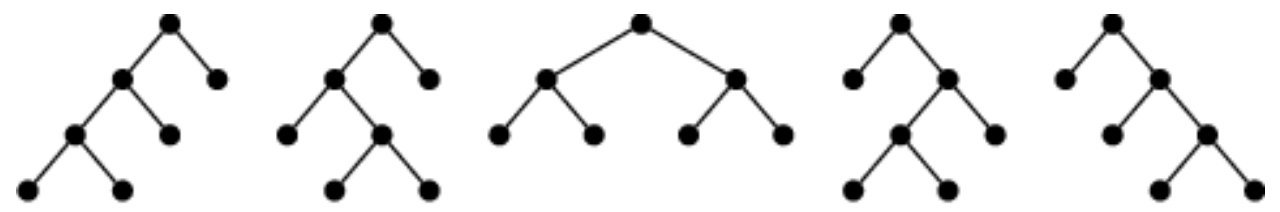

$\mathcal{C}_{n}$ is the number of colouring of the full binary trees of $2 n+1$ vertices with colours black and white, such that root is black, the right child of any vertex and the leaves are white [FMP]:
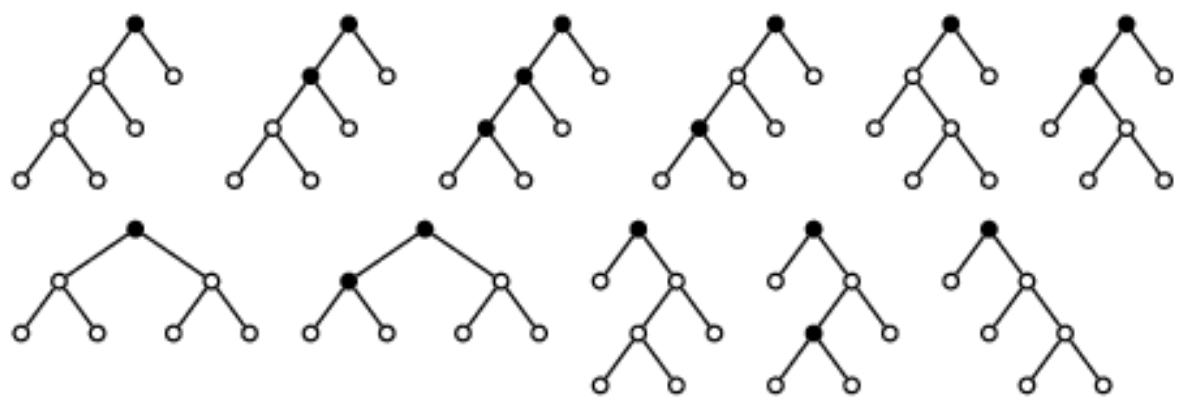

3. $C_{n}$ is the number of non-crossing partitions of the set $\{1, \ldots, n\}$ :
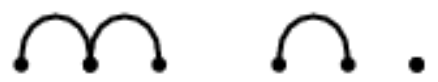

$\begin{array}{lll}1 & 2 & 3\end{array}$

$\begin{array}{lll}1 & 2 & 3\end{array}$
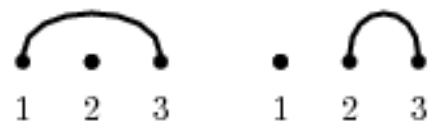

$\begin{array}{lll}1 & 2 & 3\end{array}$

123 
$\mathcal{C}_{n}$ is the number of non-crossing partitions of $\{1, \ldots, n\}$ such that each arc can be solid or dotted FMP:
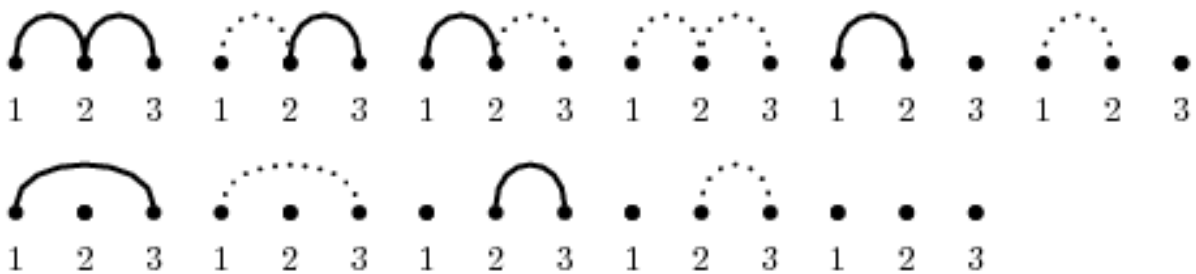

4. $C_{n}$ is the number of the non-crossing two-partitions of the set $\{1, \ldots, 2 n\}$ :

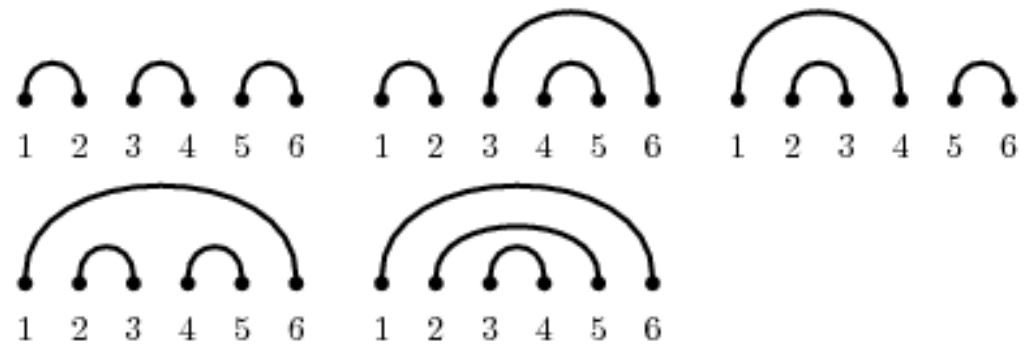

$\mathcal{C}_{n}$ is the number of the non-crossing two-partitions of the set $\{1, \ldots, 2 n\}$ such that nearest neighbour arcs $(i, i+1)$ except the leftmost one are bold or dotted, while all other arcs remain uncoloured [FMP]:
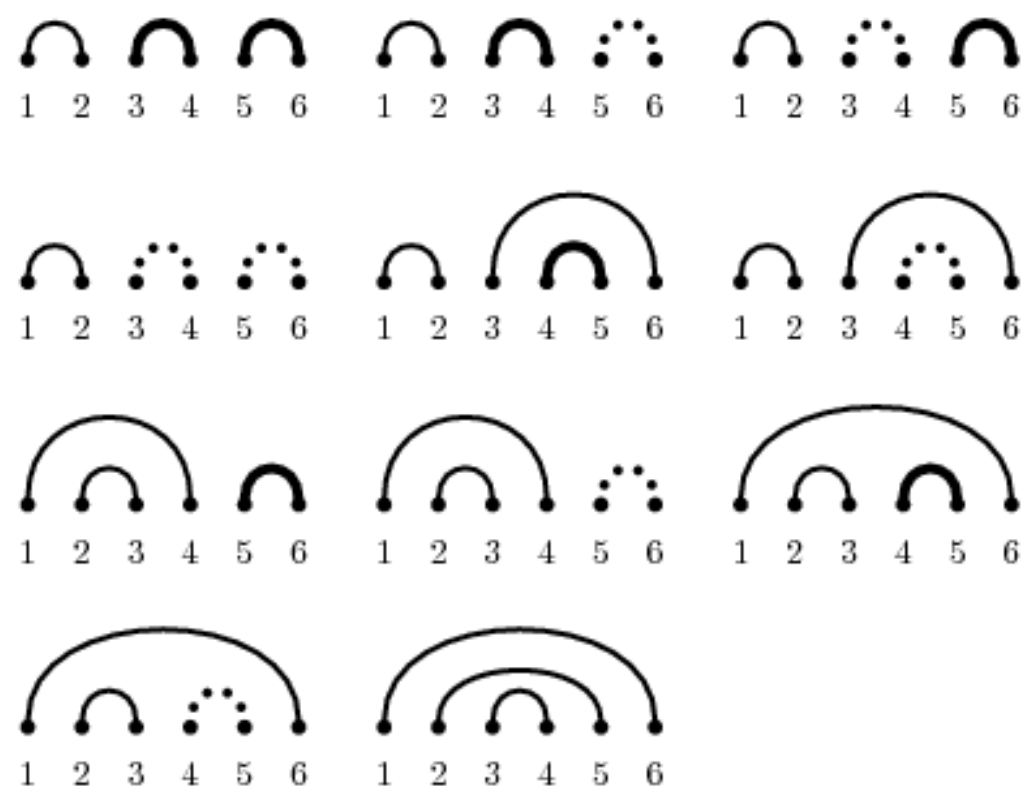
4. The super-Catalan measure. In [LR1, LR2] it was shown that the generating function of $\mathcal{C}_{n}$ for $x$ in some neighbourhood $U$ of 0 is equal to

$$
M_{\nu}(x)=\sum_{n=0}^{\infty} \mathrm{e}_{n} x^{n}=\frac{1+x-\sqrt{1-6 x+x^{2}}}{4 x},
$$

where the square root is understood in the natural way.

We know that the Cauchy transform of a compactly supported measure $\nu$ is related to the generating function of moments for complex $z$ in a neighbourhood of 0 by

$$
M_{\nu}(z)=\frac{1}{z} G_{\nu}\left(\frac{1}{z}\right) .
$$

We know the function $M_{\nu}(x)$ and need to find the Cauchy transform $G_{\nu}(z)$. To this end we need to find a suitable extension of $M_{\nu}(x)$ for our super-Catalan measure given by the formula (4.1) to a neighbourhood $0 \in U \subset \mathbb{C}$. To do so, we need to carefully specify the branch of the square root. Since $1-6 z+z^{2}$ maps $0 \in U \subset \mathbb{C}$ into $1 \in V \subset \mathbb{C}$, we need to take the branch cut for instance along the negative real numbers and assume $\sqrt{1}=1$.

Thus, we have

$$
G_{\nu}(z)=\frac{1}{z} M_{\nu}\left(\frac{1}{z}\right)=\frac{1+z-z \sqrt{\frac{1-6 z+z^{2}}{z^{2}}}}{4 z},
$$

where the square root is the one chosen for $M_{\nu}(z)$. The Stieltjes inversion formula allows us to find out the actual measure $\nu$ :

$$
\nu=\frac{1}{2} \delta_{0}+\frac{\sqrt{6 x-1-x^{2}}}{4 \pi x} \mathbb{1}_{[3-2 \sqrt{2}, 3+2 \sqrt{2}]}(x) \mathrm{d} x .
$$

The function $G_{\nu}^{-1}(z)$ on a neighbourhood of 0 is easily seen to be

$$
G_{\nu}^{-1}(z)=\frac{z-1}{z(2 z-1)} .
$$

Thus, the Voiculescu $R^{\boxplus}$-transform of $\nu$ is

$$
R_{\nu}^{\boxplus}(z)=\frac{1}{1-2 z}=\sum_{n=0}^{\infty} 2^{n} z^{n} .
$$

Hence, the free cumulants of $\nu$ are equal to

$$
r_{\nu}(n)=2^{n-1},
$$

and because this series is positive definite, the measure $\nu$ is freely infinitely divisible.

5. The symmetric super-Catalan measure. It turns out that in order to get some interesting relationships between the Catalan and super-Catalan numbers, we need to work not with the measure $\nu$ but with a measure $\mu$ such that its odd moments $m_{\mu}(2 k+1)=0$ and even moments $m_{\mu}(2 k)=m_{\nu}(k)=\mathcal{C}_{k}$.

With a reasoning similar as above, we get that the extension $M_{\mu}(z)$ analytic on some $0 \in U \subset \mathbb{C}$ has the form

$$
M_{\mu}(z)=\frac{1+z^{2}}{4 z^{2}}-\frac{1}{4 z^{2}} \sqrt{1-6 z^{2}+z^{4}}
$$


where again the branch cut is taken along the negative real numbers and $\sqrt{1}=1$. It means that the Cauchy transform of $\mu$ is equal to

$$
G_{\mu}(z)=\frac{1+z^{2}}{4 z}-\frac{z}{4} \sqrt{\frac{1-6 z^{2}+z^{4}}{z^{4}}} .
$$

The continued fraction form of the Cauchy transform of $\mu$ is equal to

$$
G_{\mu}(z)=\frac{1}{z-\frac{1}{z-\frac{2}{z-\frac{1}{z-\frac{2}{z-\ddots} .}}},}
$$

the Jacobi coefficients are equal to $\lambda_{2 n}=2, \lambda_{2 n+1}=1$. Such a measure was obtained as a Gaussian measure for the $r$-free convolution [B, BKW, $\mathrm{KY}]$ :

$$
\mu=\frac{1}{2} \delta_{0}+\frac{1}{2 \pi|x|} \sqrt{8-\left(x^{2}-3\right)^{2}} \mathbb{1}_{[-\sqrt{2}-1,-\sqrt{2}+1] \cup[\sqrt{2}-1, \sqrt{2}+1]}(x) \mathrm{d} x .
$$

Let us also note $[\mathrm{KY}]$ that we can calculate the $2 n$-th moment of $\mu$, that is

$$
\mathrm{C}_{n}=\sum_{k=0}^{n} \frac{1}{n}\left(\begin{array}{l}
n \\
k
\end{array}\right)\left(\begin{array}{c}
n \\
k+1
\end{array}\right) 2^{k}
$$

Let us calculate the Voiculescu $R^{\boxplus}$-transform of $\mu$. By Proposition 8.1 of [BV] it is enough to define $G_{\mu}^{-1}(z)$ on a neighbourhood of 0 . Thus, since

$$
G_{\mu}^{-1}(z)=\frac{2 z^{2}+1+\sqrt{1+4 z^{4}}}{2 z}
$$

where the natural branch of the square root is cut along the negative real numbers, we have

$$
R_{\mu}^{\boxplus}(z)=G_{\mu}^{-1}(z)-\frac{1}{z}=\frac{2 z^{2}-1+\sqrt{1+4 z^{4}}}{2 z} .
$$

Using the well-known formula for small $z$

$$
\sum_{n=0}^{\infty} C_{n} z^{n}=\frac{1-\sqrt{1-4 z}}{2 z}
$$

where $C_{n}$ are the Catalan numbers, we obtain

$$
-\frac{1-\sqrt{1+4 z^{4}}}{2 z}=z^{3} \frac{1-\sqrt{1-4\left(-z^{4}\right)}}{2\left(-z^{4}\right)}=z^{3} \sum_{n=0}^{\infty} C_{n}\left(-z^{4}\right)^{n} .
$$

It means that

$$
R_{\mu}^{\boxplus}(z)=\frac{2 z^{2}-1+\sqrt{1+4 z^{4}}}{2 z}=z+z^{3} \sum_{n=0}^{\infty} C_{n}\left(-z^{4}\right)^{n}=z+\sum_{n=0}^{\infty}(-1)^{n} C_{n} z^{4 n+3} .
$$

Thus the free cumulants of $\mu$ are equal to

$$
r_{\mu}(2)=1, \quad r_{\mu}(4 n+4)=(-1)^{n} C_{n}, \quad r_{\mu}(2 n+1)=r_{\mu}(4 n+2)=0 .
$$

Proposition 1. The measure $\mu$ is not infinitely divisible with respect to the free convolution. 
Proof. Because $\mu$ is freely infinitely divisible iff $\left\{r_{\mu}(n+2)\right\}$ is positive definite, and

$$
\operatorname{det}\left(\begin{array}{lll}
1 & 0 & 1 \\
0 & 1 & 0 \\
1 & 0 & 0
\end{array}\right)=-1<0
$$

we deduce that $\mu$ is not freely infinitely divisible.

Speicher $[\mathrm{Sp}$ discovered the moment-cumulant formulae for the free convolution of Voiculescu

$$
m_{\nu}(n)=\int x^{n} d \nu(x)=\sum_{\pi \in \mathrm{NC}(n)} r_{\nu}(\pi)=\sum_{\substack{\pi \in \mathrm{NC}(n) \\ \pi=\left\{B_{1}, \ldots, B_{k}\right\}}} \prod_{i=1}^{k} r_{\nu}\left(\left|B_{i}\right|\right),
$$

where $\mathrm{NC}(n)$ is the set of non-crossing partitions of the set $\{1, \ldots, n\}$. In our case we will only consider non-crossing two-partitions and non-crossing partitions with the numbers of elements in each block divisible by 4 . Thus we have

Proposition 2.

$$
\mathcal{C}_{n}=\sum_{\substack{\pi \in \mathrm{NC}(2 n) \\ \pi=\left\{B_{1}, \ldots, B_{k}\right\} \\\left|B_{i}\right|=2 \vee\left|B_{i}\right|=4 m}} \prod_{i=1}^{k} r_{\mu}\left(\left|B_{i}\right|\right),
$$

with $r_{\mu}(2)=1, r_{\mu}(4 m)=(-1)^{m-1} C_{m-1}$.

On the other hand, we can use the moment-cumulant formula proven in $[\mathrm{KY}]$. Let us remind some definitions.

Definition 1. For a block $B=\left\{b_{1}, b_{2}, \ldots, b_{|B|}\right\} \in \pi$ with $b_{1}<b_{2}<\ldots<b_{|B|}$ of a non-crossing partition $\pi$ define $\mathrm{uc}(B):=\#\left\{b_{i} \in B: i>1\right.$ and $\left.b_{i} \neq b_{i-1}+1\right\}$, where \# means the cardinality. For $\pi$ define $\mathrm{uc}(\pi)=\sum_{B \in \pi} \mathrm{uc}(B)$, number of uncontractive arcs of the partition $\pi$.

Thus we have

Proposition 3.

$$
\bigodot_{n}=\sum_{\pi \in \mathrm{NC}_{2}(2 n)} 2^{\mathrm{uc}(\pi)}
$$

We can also use a different statistics on non-crossing pair partitions. Let $\operatorname{eb}(\pi)$ be the number of blocks of even depth in non-crossing pair partition $\pi$. Here the depth $\mathrm{d}_{\pi}\left(B_{i}\right)$ of the block $B_{i}$ of the non-crossing partition $\pi$ is defined by

$$
\mathrm{d}_{\pi}\left(B_{i}\right)=\#\left\{B_{j} \mid B_{j} \supset B_{i}\right\} .
$$

Proposition 4. The statistics $\mathrm{eb}(\pi)$ and $\mathrm{uc}(\pi)$ are equidistributed on $\mathrm{NC}_{2}(2 n)$, that is,

$$
\mathcal{C}_{n}=\sum_{\pi \in \mathrm{NC}_{2}(2 n)} 2^{\mathrm{eb}(\pi)}=\sum_{\pi \in \mathrm{NC}_{2}(2 n)} 2^{\mathrm{uc}(\pi)} \quad(n \geq 1) .
$$

Let us remind the classical definition of the Kreweras complement:

Definition 2. Let $\pi_{1}$ and $\pi_{2}$ be two partitions in $\mathrm{NC}(n)$. By the transformation $i \mapsto 2 i-1$ we can copy $\pi_{1}$ to the set $\{1,3,5, \ldots 2 n-1\}$ and copy $\pi_{2}$ to $\{2,4,6, \ldots 2 n\}$ by the transformation $i \mapsto 2 i$. Combining the two partitions, we obtain a partition of $2 n$ 
elements, which will be denoted by $\pi_{1} \sqcup \pi_{2}$. Given $\pi \in \mathrm{NC}(n)$, there is a largest among partitions $\pi^{\prime} \in \mathrm{NC}(n)$ such that $\pi \sqcup \pi^{\prime} \in \mathrm{NC}(2 n)$. We will denote this partitions by $\mathrm{K}(\pi)$ and call it the Kreweras complement of $\pi$.

Using that notion, Hiai and Petz $[\mathrm{HP}$ showed that we have the following inverse of the moment-cumulant formula:

$$
r_{\mu}(n)=\sum_{\pi \in \mathrm{NC}(n)} \prod_{B \in \pi} m_{\mu}(|B|) \prod_{D \in \mathrm{K}(\pi)}(-1)^{|D|-1} C_{|D|-1} .
$$

For our measure we obtain

Proposition 5.

$$
(-1)^{n-1} C_{n-1}=\sum_{\pi \in \mathrm{NC}(4 n)} \prod_{\substack{B \in \pi \\|B| \text { is even }}} \mathcal{C}_{|B| / 2} \prod_{D \in \mathrm{K}(\pi)}(-1)^{|D|-1} C_{|D|-1} .
$$

\section{References}

[Ai] M. Aigner, Catalan and other numbers: a recurrent theme, in: Algebraic Combinatorics and Computer Science, Springer Italia, Milan, 2001, 347-390.

[Ak] N. I. Akhiezer, The Classical Moment Problem and some Related Questions in Analysis, Oliver \& Boyd, Edinburgh-London, 1965.

[BV] H. Bercovici, D. Voiculescu, Lévy-Hinčin type theorems for multiplicative and additive free convolution, Pacific J. Math. 153 (1992), 217-248.

[B] M. Bożejko, Deformed free probability of Voiculescu, Sūrikaisekikenkyūsho Kōkyūroku 1227 (2001), 96-113.

[BKW] M. Bożejko, A. D. Krystek, Ł. J. Wojakowski, Remarks on the $r$ and $\Delta$ convolutions, Math. Z. 253 (2006), 177-196.

[Ch] T. S. Chihara, An Introduction to Orthogonal Polynomials, Mathematics and its Applications 13, Gordon and Breach, New York, 1978.

[C] L. Comtet, Advanced Combinatorics: The Art of Finite and Infinite Expansions, Reidel, Dordrecht, 1974.

[FMP] A. N. Fan, T. Mansour, S. X. M. Pang, Elements of the sets enumerated by superCatalan numbers, preprint.

[HP] F. Hiai, D. Petz, The Semicircle Law, Free Random Variables and Entropy, Math. Surveys Monogr. 77, Amer. Math. Soc., Providence, RI, 2000.

[KY] A. Krystek, H. Yoshida, The combinatorics of the $r$-free convolution, Infin. Dimens. Anal. Quantum Probab. Relat. Top. 6 (2003), 619-627.

[LR1] J.-L. Loday, M. Ronco, On the structure of cofree Hopf algebras, J. Reine Angew. Math. 592 (2006), 123-155.

[LR2] J.-L. Loday, M. Ronco, Combinatorial Hopf algebras, in: Quanta of Maths, Clay Math. Proc. 11, Amer. Math. Soc., Providence, RI, 2010, 347-383; arXiv:0810.0435v2.

[Sp] R. Speicher, Multiplicative functions on the lattice of non-crossing partitions and free convolution, Math. Ann. 298 (1994), 611-628.

[St] R. P. Stanley, Enumerative Combinatorics, vol. 2, Cambridge Stud. Adv. Math. 62, Cambridge Univ. Press, 1999.

[V] I. Vardi, Computational Recreations in Mathematica, Addison-Wesley, Redwood City, CA, 1991. 